\title{
Periodic water, interwater interval, and adjunctive behavior in a 24-hour multiresponse environment
}

\author{
WILLIAM TIMBERLAKE and GARY A. LUCAS \\ Indiana University, Bloomington, Indiana
}

\begin{abstract}
To examine the generality of the interreward response effects shown by rats under periodic food delivery, we presented $.10 \mathrm{ml}$ of water at minimum interwater intervals that ranged from 8 to $512 \mathrm{sec}$. Use of a 24-h multiresponse environment allowed evaluation of interdrink responses with respect to their excessiveness, patterning, and functional relationship to the interwater interval. In contrast to the extensive activity-inducing effects of periodic food, the only major excitatory effect of periodic water was increased attention to the water source. Although there were a few bitonic and direct relationships between interwater interval and changes in responding, the great majority of functions were inverse or inconsistent. Further, unlike the increase in drinking under periodic food, total eating decreased under periodic water. The major similarity with food reward was the apparent separation of interreward behavior into three general classes of reward-appropriate foraging responses: area-restricted search after reward, more general search (and waiting), and focal search preceding the next reward delivery.
\end{abstract}

Thirty years after Falk (1961) called attention to interreward adjunctive behavior in the form of excessive postpellet drinking under periodic food delivery, the characteristics and causation of such behavior remain controversial (Reid \& Staddon, 1986; Riley \& Wetherington, 1989; Roper, 1981; Wetherington, 1982). Among the points of contention are the following: to what extent adjunctive responses are excessive, whether the relationship between adjunctive responding and reward frequency is direct or bitonic (Reid, Vazquez, \& Rico, 1985), the number and nature of classes of adjunctive behavior, and whether adjunctive responding is similar under both food and water rewards (Rachlin \& Krasnoff, 1983; Reid et al., 1985; Wetherington \& Riley, 1985). This paper examines how these issues apply to a wide variety of interreward responses in a 24-h environment.

A major difficulty with the assessment of adjunctive behavior has been the lack of a precise definition of excessiveness. Despite extensive debate, there is continued disagreement over the proper comparison condition (see Falk, 1971; Roper, 1981; Staddon, 1977; Timberlake, 1982). Another difficulty is the nature of the relationship between reward frequency and adjunctive responding. Although a bitonic relationship between reward frequency and total adjunctive responding is well supported (e.g, Falk, 1969, 1971, 1977; Wetherington, 1982), recent investigators have focused on a direct relationship between re-

This research was supported by NIMH Grant MH37892. The authors wish to thank Hillary Case for her extensive help with data analysis and Andy Delamater for his comments. Correspondence should be addressed to William Timberlake, Psychology Department, Indiana University, Bloomington, IN 47405. ward frequency and the interreward rate of responding (i.e., response rate is highest at the shortest interreward intervals-see Killeen, Hanson, \& Osborne, 1978; Staddon, 1977; Wetherington, 1979).

It is worth pointing out that bitonic and direct relationships between different response measures and reward frequency need not be incompatible; the different measures and relationships may reflect different characteristics of the expression of adjunctive behavior in time. $\mathbf{A}$ given response may show either, both, or neither of these relationships. A direct relationship between rate of responding and reward frequency over short interreward intervals typically means that the response competes differentially well for expression in the limited time available between rewards. At the shortest intervals, such competitive responses occupy nearly the whole of the interval, resulting in a high rate of expression during the interval. As the interval increases, other responses begin to be expressed, decreasing the interinterval rate. In contrast, a bitonic relationship between total responding and reward frequency shows that the response determinants combine to continue to increase total output as the intervals get longer, although only to a point.

A final difficulty is the disagreement over the number and characteristics of classes of adjunctive responding. Staddon (1977) distinguished two groupings-interim and facultative responses. Interim responses are incentive related, are excessive, and tend to occur closely following reward. Facultative responses are unrelated to the incentive, are not excessive, and fill in the middle of the interreward interval, expanding and contracting with the interval size. Thus, drinking is classed as an interim response, whereas wheelrunning is facultative. 
Cohen, Looney, Campagnoni, and Lawler (1985; see also Campagnoni, Lawler, \& Cohen, 1986) redivided adjunctive behavior into a time-locked class and a generalactivity class. Time-locked responses (which we will refer to here as time-bound responses) are more repetitive and reflexive and occur at a fixed time following feeding, such as in drinking and conspecific attack. General-activity responses are less reflexive and expand to fill the interreward interval, such as in wheelrunning. Lucas, Timberlake, and Gawley (1988) combined aspects of both schemes with a category of focused anticipatory responses in viewing interfood behavior as the expression of a cycle of foraging. In this view, responding immediately after ingestion constitutes time-bound, area-restricted search and is followed by the transitional responses of drinking and rearing. Search behavior then expands, becoming much less focused on the food location, before narrowing again and becoming directed at the food location as the next food delivery approaches.

The use of a 24-h environment with many available responses (Lucas et al., 1988) has several advantages for the study of adjunctive behavior (see also Petersen \& Lyon, 1978). First, measurement of free responding provides an undisturbed, complete, and "ecologically fair" baseline for determining the excessiveness of responding under subsequent intermittent reward (see Roper, 1981). There are periods of responding and of not responding, and, as in the case of a foraging animal, multiple meals are embedded in a 24-h context. Second, measuring response totals across the full $24 \mathrm{~h}$ differentiates responses that are increased by periodic food delivery from responses that are simply redistributed around (or away from) food intake without changing their total occurrence. Third, a free-baseline condition can be used to establish the pattern and probability of responses preceding and following unconstrained feeding bouts (meals). By comparing the conditional response probabilities per unit time preceding and following a baseline meal with the same conditional probabilities preceding and following individual pellets, it is possible to distinguish changes in the amount of responding caused by changes in the patterning of responding from changes caused by the greater number of discrete ingestion episodes (opportunities) available when ingestion opportunities are separated in time. It is also possible to use conditional response probabilities to provide an estimate of local excessiveness that is not biased by changes in the amount of time that is available for responding. Fourth, the variety of possible responses, including eating, drinking, restless activity, wood chewing, rearing, wheelrunning, and nesting, allows an extensive examination of the classification systems proposed by Staddon (1977), Cohen et al. (1985), and Lucas et al. (1988).

The present study used the advantages of a 24-h multiresponse environment to study responding during periodic presentations of water. Despite the extensive work on adjunctive behavior, there has been relatively little at- tention paid to interreward responses other than drinking and to rewards other than food (Roper, 1981). The contrast of the present results based on water with those of Lucas et al. (1988) based on food, with the same kind of environment used for both studies, should provide insight into both the general and specific determinants of adjunctive and anticipatory behavior.

An intuitive prediction based on the assumption of general functional laws of behavior is that periodic water should produce the same activating effects on interreward responding that occur with periodic food, with eating symmetrically replacing drinking as a dominant adjunctive response. However, if a key to interreward responding is its relation to foraging cycles, important differences are likely. Several types of studies have shown less active anticipation of water than of food. Campbell and Cicala (1962) and Hall (1955) found that water deprivation produced little or no increase in general activity. McDonald and de Toledo (1974) showed that there was no active frustration reaction to the absence of a water reward (as opposed to food) in two partial reinforcement paradigms. Reberg, Mann, and Innis (1977), using a 60-sec interreward interval and choices of food or water, reported that rats showed much less gross locomotor activity in interwater intervals than in interfood intervals.

Extrapolating from these results, there should be little, if any, increase in active general search responses during the interwater interval. Instead, the animals are more likely to remain close to the water source. Results from previous studies of periodic water reward that used shorter sessions and fewer intervals and responses generally support this prediction. Reid et al. (1985) found that only one response markedly increased under periodic water access, namely, responding directed at the water source after and especially before delivery of water. At interwater intervals of 90 and $180 \mathrm{sec}$, there was an indication of increased grooming and eating for some animals, although at intervals below $90 \mathrm{sec}$, eating and grooming were below baseline. Sniffing and rearing appeared depressed with respect to baseline at all intervals. An exception to this picture of decreased activity was the report by King (1974) that wheelrunning increased under periodic water delivery.

A number of studies focusing on the effects of periodic water on feeding showed either no increase or a slight decrease in intake (Carlisle, Shanab, \& Simpson, 1972; King, 1974; Myerson \& Christiansen, 1979; Rachlin \& Krasnoff, 1983; Wetherington \& Brownstein, 1979; Wetherington \& Riley, 1985). The last two studies emphasized that although eating was delayed in the interwater intervals, it took place at a time that was relatively proportional to the length of the interval. An exception to these results was provided by Bellingham, Wayner, and Barone (1979), who found a near doubling of the amount of feeding under an FT 1-min water schedule over a home cage control receiving the same amount of water in massed form. None of these studies provided solid evidence of a bitonic or a direct relationship between the interwater interval and 
either the total or conditional probability of eating or any other response (although perhaps the intervals used were too short; see Wetherington \& Brownstein, 1979).

In sum, the present study examined the effects of periodic water presentation on behavior in a multiresponse 24-h environment. We used an extended range of interwater intervals, from 8 to $512 \mathrm{sec}$. The results were compared to the previously reported effects of periodic water in shorter sessions and to the effects of periodic food in the same 24-h environment (Lucas et al., 1988). The use of the 24-h environment allowed convergent ways of measuring excessive behavior, including both daily response totals and conditional probabilities of responding within episodic feeding bouts. The results separated excessiveness from simple rearrangement of responding and distinguished changes in the probability of responding per opportunity from changes in the number of ingestions or ingestion bouts produced by the schedule. Finally, the use of a variety of responses allowed extensive examination of the temporal patterning and classes of interwater responding.

\section{METHOD}

\section{Subjects}

Four female Sprague-Dawley-derived albino rats were obtained from the local breeding colony. After weaning, the rats were housed socially until placed in the experimental chambers at 90 days of age.

\section{Apparatus}

The test chamber was $30 \times 30 \times 30 \mathrm{~cm}$. On the front wall were a feeder, a water dispenser, two retracted levers, and a plate for sensing rearing; a sniff hole, wood block, nest, and rearing-plate were on the back wall; and there was access to a running wheel on a side wall (see Figure $l$ in Lucas et al., 1988). Movement of the block was monitored by contact sensors located in the base; sniffing was detected by a photocell located on the outside of the hole; rearing was detected by continuity circuits involving the floor and the rearing plate. The floor was constructed of stainless $3 / 16$-in.diam rods placed ${ }_{h}$ in. on center and consisted of two frames pivoted at $90^{\circ}$ from each other to allow recording of whichever quadrant the animal was in. A covered nest and a wheel were accessible from the chamber. A microswitch on the nest floor determined when the rat was in the nest. The rat's presence in the wheel was based on the absence of the rat from the nest or the chamber floor, whereas complete wheel revolutions were determined by a photoreceptor and a small "flag" attached to the rim of the wheel.

The water dispenser consisted of a $1.0-\mathrm{cm}$-diam brass bowl into which two $.05-\mathrm{ml}$ drops of water were pulsed in succession with audible clicks to provide the $.10-\mathrm{ml}$ reward that was used in the present experiment. Drinking all of the water broke a continuity circuit so that the computer could be programmed to replace the water at any chosen interval. The food-pellet dispenser worked in the same general way, except that detection of the presence of the pellet was performed by a photobeam. The presence of the animal's head in either the food or water openings also was detected by a photocell. The food and water dispensers were centered on the front wall, one on either side of one of the axes of the tilt floor. An 8-cm partition between the dispensers prevented the animal from having its head in one dispenser and its body on the opposite side. Each chamber was enclosed in a sound-attenuating metal box vented to the outside. The boxes were located in a dedicated room maintained at $21^{\circ} \mathrm{C}\left( \pm 1^{\circ}\right)$ and $50 \%$ humidity $( \pm 15 \%)$. A 12:12-h light:dark cycle was always in effect. A more complete description of the apparatus is available in Lucas et al. (1988).
Table 1

Description of Response Categories

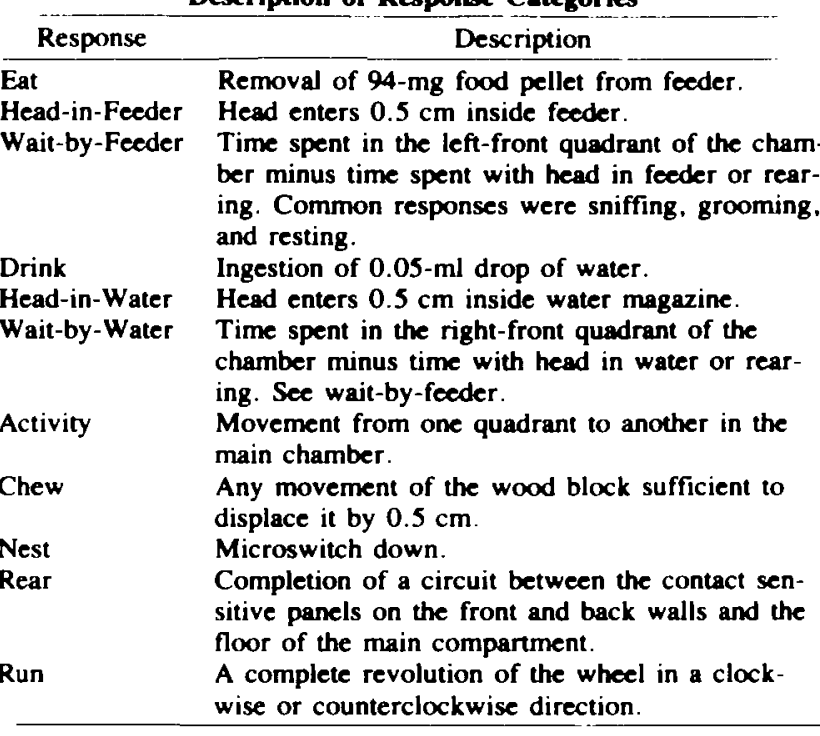

\section{Procedure}

The rats were weighed during a daily maintenance period in the light part of the cycle. The rats were confined to their nest, and the entire nest was weighed to avoid disrupting the animals by handling. The nest doors opened 15 min after all maintenance had ended to minimize any effects of the disturbance on subsequent behavior in the chamber. Following a 40-day baseline period of unconstrained access to all responses in the chamber, access to water was constrained by imposing a minimum interval between the drinking of $.10 \mathrm{ml}$ of water and the receiving of the next delivery. These minimum intervals ranged from 8 to $512 \mathrm{sec}$ in an ascending series. Each constraint was in effect for 12 successive days and was followed by a return to unconstrained baseline conditions.

Control of and data recording from each chamber were arranged by an IBM PC located in a separate room and running Spyder Systems' Conman software (Bloomington, IN) operating at 0.1-sec resolution. A 32-channel event record synthesized in software for each chamber was stepped each $4 \mathrm{sec}$. The presence or absence of $\alpha$ currence of each behavior was recorded in 4-sec bins. In addition, the absolute counts of the number of pellets, .05-ml drinks, and wheel turns were recorded separately in 30 -min bins.

The responses used in this study are shown in Table 1. Although wait-by-feeder and wait-by-water are defined in part by the absence of other behavior while the animal was in the feeder or water quadrant, occasional direct observation of the animals revealed a reasonably coherent category. The rats spent these periods sniffing the floor, grooming, and resting near the water or feeder.

A log-survivor analysis (Lehner, 1979) was used to determine the size of the interdrink interval that marked the end of a bout of drinking. A large discontinuity in the distribution of cumulative survivors occurred at approximately $1 \mathrm{~min}$; therefore, the animals in both the baseline and contingency conditions were considered to be within a bout of drinking, so long as they continued to drink within $1 \mathrm{~min}$ of the time water was made available.

\section{RESULTS}

\section{Excessiveness and the Effects of Reward Frequency}

Table 2 shows the asymptotic mean daily body weight and the mean daily total bins during which a response occurred for each animal during the last 4 days of both base- 
Table 2

Mean Daily Totals in the First Baseline by Responses and Subjects and the Percent of First Basetine Responding Across the Other Conditions

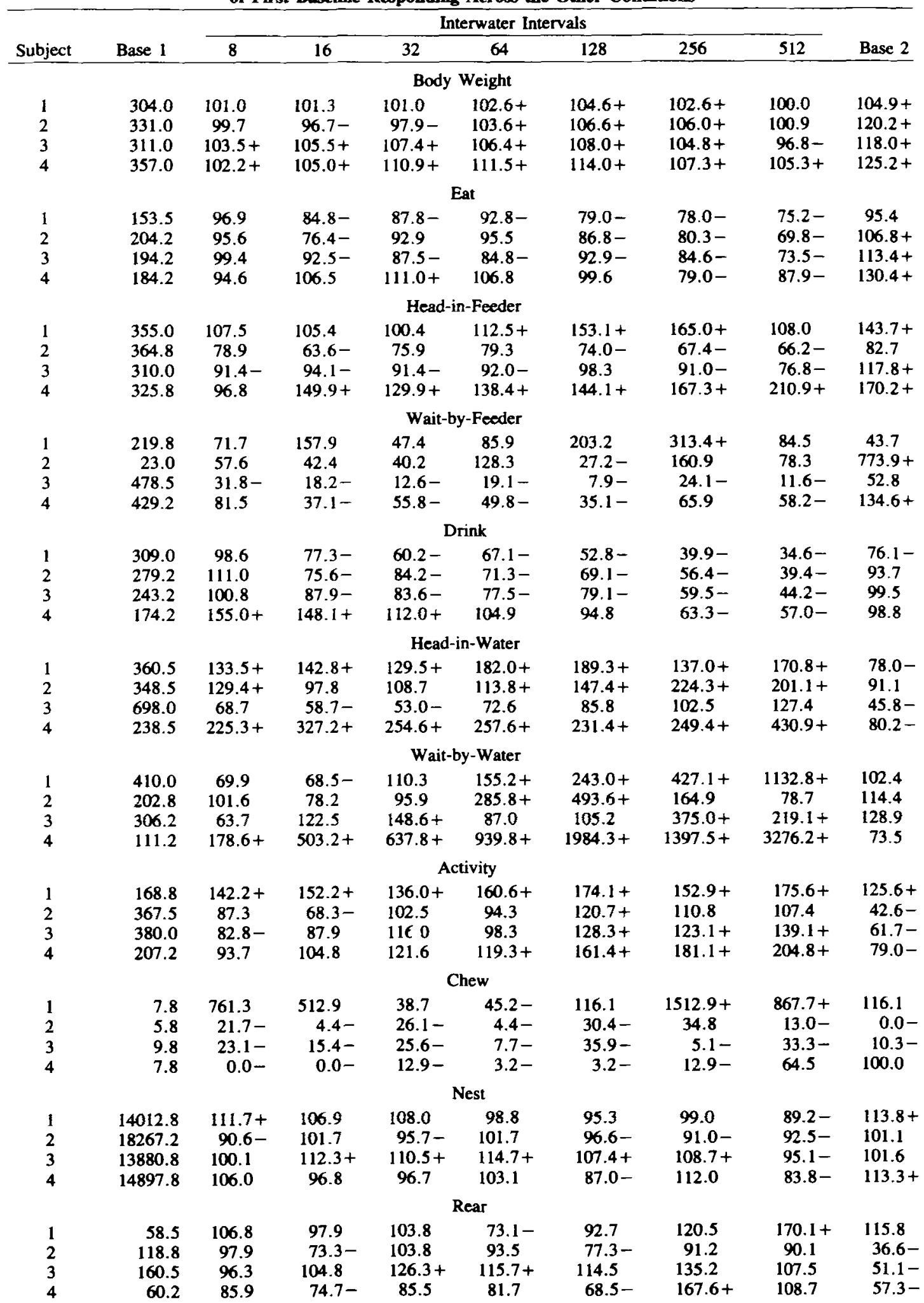




\begin{tabular}{|c|c|c|c|c|c|c|c|c|c|}
\hline \multirow{3}{*}{ Subject } & \multirow[b]{3}{*}{ Base 1. } & & & \multicolumn{3}{|c|}{ Table 2 (Continued) } & \multirow{3}{*}{256} & \multirow{3}{*}{$512-$} & \multirow[b]{3}{*}{ Base 2} \\
\hline & & & & \multicolumn{3}{|c|}{ Interwater Intervals } & & & \\
\hline & & 8 & 16 & 32 & 64 & 128 & & & \\
\hline \multicolumn{10}{|c|}{ Run } \\
\hline 1 & 349.0 & 77.2 & 84.4 & $74.5-$ & 91.4 & $70.1-$ & 83.2 & 75.0 & 68.9 \\
\hline 2 & 694.8 & $289.1+$ & $169.1+$ & $187.1+$ & $60.0-$ & $50.8-$ & $144.7+$ & $154.7+$ & 106.4 \\
\hline 3 & 1229.8 & 114.0 & 93.3 & $126.8+$ & 112.3 & $133.9+$ & 95.8 & $191.0+$ & 129.4 \\
\hline 4 & 230.5 & 68.8 & $55.1-$ & $56.5-$ & $28.0-$ & $21.6-$ & $35.6-$ & $37.6-$ & $17.1-$ \\
\hline
\end{tabular}

Note-Except for body weight (in grams), the first baseline data are reported as the number of 4-sec bins per day $(21,600$ possible bins) in which the response occurred, averaged over the final 4 days of each condition. A plus or minus sign after an entry indicates the direction of a significant change of $2 S E M$ from the initial baseline.

line conditions and all interwater interval conditions. The initial baseline is presented as the absolute number of 4 -sec bins in which a behavior was recorded. The remaining data are expressed as percentages of that baseline. The presence of a plus or minus sign next to an entry indicates the direction of a significant change of $2 S E M$ from the initial baseline. Figure 1 shows another view of the data, plotting the log percentage intervals of occurrence for selected responses averaged over all 4 animals at each condition.

The data show little evidence of excessive daily responding under periodic presentation of water and essentially no support for bitonic relations between total responding and reward frequency. Both wait-by-water and head-in-water showed sizable and consistent increases over baseline levels. Activity showed a slight but consistent increase with interval size. Rear, run, chew, nest, and feeder-related responses showed inconsistent changes over animals and intervals, except for a reduction in nest at the 512-sec interwater interval. Eating and drinking also decreased significantly at the longer interwater intervals for all animals, although without producing a systematic effect on body weight or on feeder-related responding.

Although these data clearly anchor the definition of excessiveness in daily response totals, they do not distinguish response changes that occur within a drinking bout from those that occur between drinking bouts at some temporal and motivational distance. This distinction may be important in understanding changes in responding occurring in shorter sessions (typically based on one ingestion bout). For the present analysis, a bout of drinking was defined as that period of time during which an animal continued to drink all available water "pellets" within $1 \mathrm{~min}$ of their delivery.

As shown in Figure 2, the percentage of daily responding shown in Table 2 and Figure 1 that occurred within a bout of drinking markedly increased as the interwater interval increased. By the 32-sec interval, most daily headin-water and wait-by-water responses occurred within drinking bouts. By the 128-sec interval, most rear, activity, and run responses occurred in drinking bouts. Finally, by the 512 -sec interval, most eating occurred within drinking bouts.

It is worth noting that a marked increase in eating within drinking bouts did not occur until nearly all time avail- able during the night part of the cycle was contained within the drinking bouts. (The maximum time available outside the drinking bouts can be approximated by multiplying the number of drinks by the minimum interwater interval specified by the schedule and subtracting the result from $720 \mathrm{~min}$, which is the duration of the night.) Finally, the increases in wait-by-water, rear, and activity were

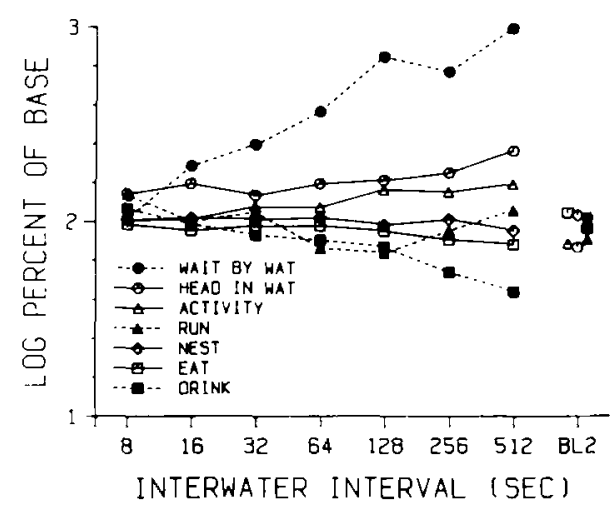

Fizure 1. Average daily total responding at asymptote (inst 4 days) in each interwater interval condition, expressed as the log percentage of baseline responding. The numeral 2 on the ordinate marks $100 \%$ of baseline.

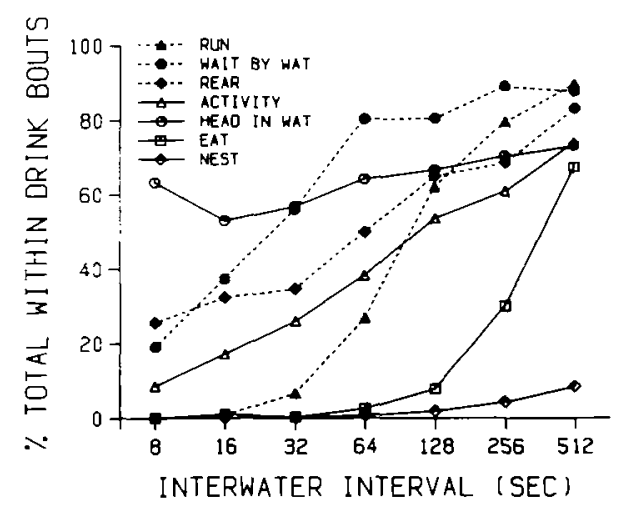

Figure 2. Average percentage of dally response totals that occurred within (as opposed to between) bouts of drinking. The mensure is shown at asymptote for each interwater interval condition. 


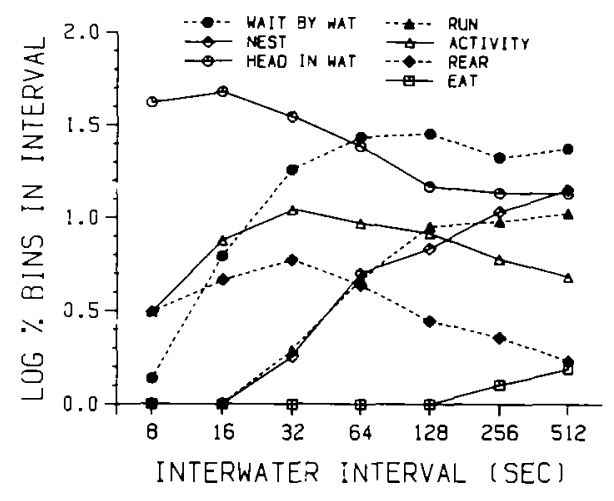

Figure 3. The average log percentage of the $4 \mathrm{sec}$ bins in interdrink intervals within a bout during which a response occurred. The numeral 2 on the ordinate marks $100 \%$ of baseline.

predominately linear under the doubling scale on the $x$-axis, implying a rate of increase of less than a constant proportion of the interval available. In contrast, the initial increases in run and eat were accelerating, implying a more proportional increase with the size of the interwater interval.

Figure 3 provides another way of looking at the changes in within-bout responding as a function of the interwater interval. This figure shows the proportion of 4-sec bins within the interwater interval during which a response $o c$ curred, averaged over animals and the last 4 days of each interwater interval. At the shorter intervals, this measure reveals the relative priority of the different responses under high levels of competition. At longer intervals, this measure reflects more the sustained motivation for a response across the entire interval. Individual analyses of variance (ANOVAs) showed that all responses plotted in Figure 3 changed significantly across interval conditions in the percentage of bins in which they occurred. However, only head-in-water shows a direct relationship between reward frequency (the inverse of the interval) and responding. Activity and rear show bitonic relationships with reward frequency, and the remainder show inverse relations.

The dominant interwater response up to the 32-sec interval was head-in-water, composed of drinking, nosing about, and digging in the water bowl. Any remaining time in these shorter intervals was spent increasingly in waitby-water, rear, and activity (often moving over to the feeder area and inserting the head but not eating). Nest and run also emerged at the 32-sec interval. At interwater intervals from 64 to $512 \mathrm{sec}$, head-in-water and wait-bywater remained the dominant responses, with the latter response occurring in better than $25 \%$ of the bins. Nest and run continued to increase, eventually occurring in more than $10 \%$ of the bins at the longest intervals. Activity decreased to about $5 \%$ of the bins. Finally, eating began to occur within bouts at intervals greater than $128 \mathrm{sec}$.

As in the case of any measure of behavior, it is important to question whether the definition of a bout of drinking used here is arbitrary, that is, unrelated to the determinants of responding. As indicated above, a bout of drinking was defined by the present criterion as all drinks that occurred within 1 min of the time water was made available. This criterion was well supported in baseline drinking by a marked discontinuity in a log-survivor function relating the frequency of interdrink intervals longer than a given interval to the size of the interval. The behavioral meaningfulness of the bout criterion was also supported under the other conditions. Comparison of the minimum interwater interval specified by the experimenter with the actual interdrink interval shown by the rats within a bout of drinking is shown in Table 3 (the terminating minute of the bout criterion was subtracted out because, by definition, it was not part of an interdrink interval falling within the bout). The average time to finish drinking after water was delivered was never longer than $11.6 \mathrm{sec}$. Furthermore, instead of increasing with the size of the interval, as might be expected if the animals were estimating the passage of time, the average time to drink after availability actually decreased at the longest interwater intervals. Taken together, these data suggest that the present bouts of drinking were well defined by an animal's readiness to drink when water became available.

Table 3 also shows the basis for the considerable decline in daily total drinking over presentation intervals, which was documented in Figure 1 and Table 2. The average number of drinking bouts per day remained relatively constant or increased slightly over presentation intervals from 8 to $128 \mathrm{sec}$ but fell rapidly at the longest intervals. In contrast, the number of water presentations per bout first decreased and then increased as the presentation intervals

Table 3

Characteristics of Daily Drinking

\begin{tabular}{|c|c|c|c|c|}
\hline \multicolumn{3}{|c|}{ Within Bout Interwater Interval (sec) } & \multirow{2}{*}{$\begin{array}{c}\text { Average No. } \\
\text { of Daily } \\
\text { Drink Bouts }\end{array}$} & \multirow{2}{*}{$\begin{array}{c}\text { Average Water } \\
\text { Presentations } \\
\text { per Drink Bout }\end{array}$} \\
\hline $\begin{array}{l}\text { Programmed } \\
\text { Minimum IWI } \\
\end{array}$ & $\begin{array}{c}\text { Average } \\
\text { Actual IWI }\end{array}$ & $\begin{array}{c}\text { Average } \\
\text { Difference }\end{array}$ & & \\
\hline 8 & 13.6 & 5.6 & 33.6 & 7.8 \\
\hline 16 & 25.6 & 9.6 & 37.2 & 5.7 \\
\hline 32 & 43.6 & 11.6 & 39.6 & 4.8 \\
\hline 64 & 74.6 & 10.6 & 35.5 & 5.0 \\
\hline 128 & 136.5 & 7.5 & 32.1 & 4.9 \\
\hline 256 & 262.3 & 6.3 & 22.1 & 5.2 \\
\hline 512 & 517.9 & 5.9 & 13.7 & 6.8 \\
\hline
\end{tabular}

Note-All averages are over last 4 days of a condition. 
lengthened and the number of bouts fell, although the increase was not enough to maintain total intake.

\section{Responding per Opportunity and Pre- and Postdrink Patterning within Bouts}

Another way to look at excessiveness is to consider the amount of responding per opportunity. An overall measure of responding per opportunity can be obtained by dividing the other daily response totals in Table 2 by the total number of drinks. It can be seen by inspection of Table 2 and Figure 1 that this computation will result in an increase in all responses as the interwater interval increases, because the amount of drinking decreases to an average of less than $50 \%$ of its baseline amount. However, for two reasons, this measure is not a good estimate of excessive responding with respect to the intermittent delivery of water. First, it does not consider the effects of the increased time available for responding resulting from the decrease in total drinking and the increase in spacing between drinks. Second, it does not distinguish between responding that is proximal to the delivery of water and responding that occurs at other points in the day.

Figure 4 shows a measure of responding per opportunity that resolves the proximity criticism by considering only responding occurring within a bout of drinking. The measure also controls for changes in the time available for responding by computing the probability of responding per constant time unit (each 4-sec bin). This measure is essentially the conditional probability of responding per 4-sec interwater bin (responses per 4-sec opportunity) associated with a bout of drinking. Figure 4 shows the temporal distribution of 10 responses both prior to and following delivery of water. For each response, each interwater interval is plotted separately as the average log percentage occurrence in each 4-sec bin over the last 4 days of each condition. The baseline response probabilities (the conditional probabilities of responding by 4-sec bins for $240 \mathrm{sec}$ before or after freely occurring drinking bouts in baseline) are shown by dotted lines.

The left-hand set of graphs shows the predrink half of the interwater and baseline distributions, and the righthand set of graphs shows the postdrink half of the interwater and baseline distributions. Separate plots were made for pre- and postdrinking behavior (rather than a single plot of the entire interwater interval), for two reasons. First, a given interwater interval only specifies the minimum period between drinks, not the actual interval. The actual interval varied over conditions, days, and animals (see Table 3). To locate responding precisely with respect to the end or the beginning of a set of intervals, it is necessary to synchronize the responses at either the end or the beginning of the interwater interval. Second, the separate pre- and postdrink plots made it easier to distinguish any time-bound responding preceding or following drinking.

\section{GROUP PRE-DRINKING BEHAVIORS}
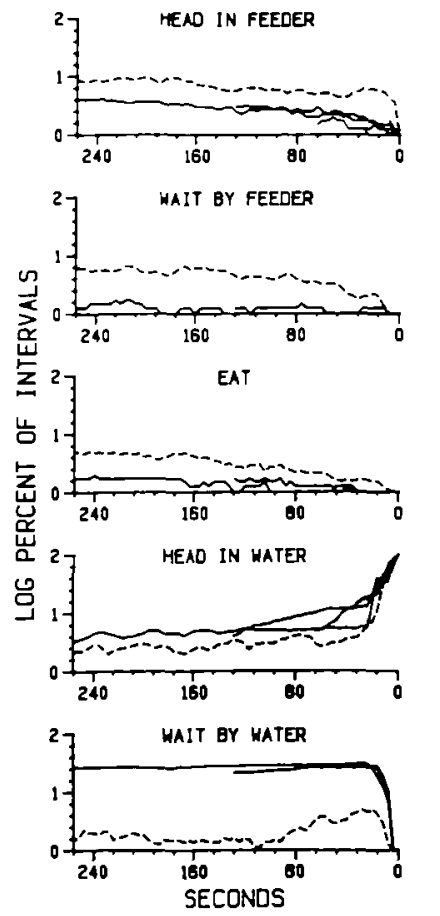
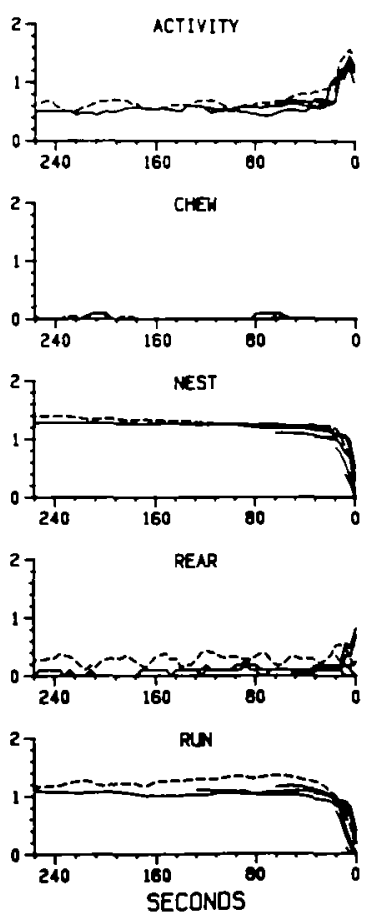

GROUP PCST-DR:NKING BEHAVIORS
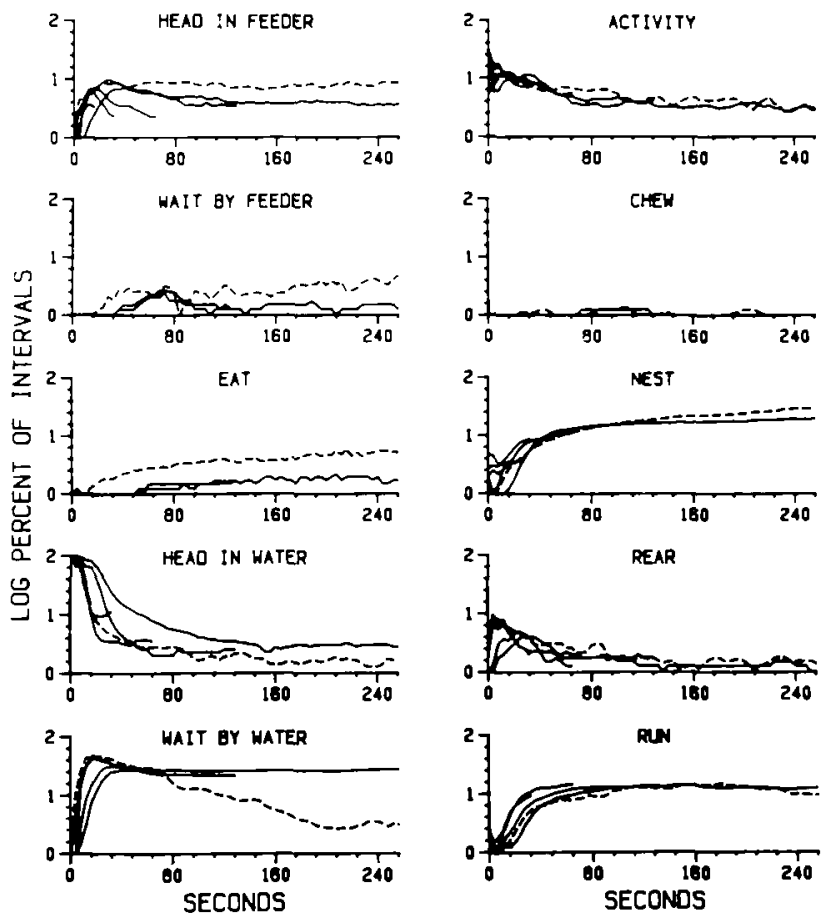

Figure 4. The distribution of responding in 4-sec bins for the half of the interfood interval preceding (predrinking) or following (postdrinking) drinking (labeled 0 sec on the $x$-axis). The solid lines show distributions under the different interwater intervals; the dashed line shows the distribution in free baseline over the same bins. The data are group averages plotted as the log of the mean percentage of total bins in which each response occurred. The numeral 2 on the ordinate marks $100 \%$ of baseline. 
An important consideration in examining these data is how to determine the reliability and significance of the indicated effects of conditions. We used two approaches. The broadest approach was to compare average responding across intervals with comparable intervals of responding in baseline. This was done in a $2 \times 6$ (condition [baseline and constraint] $\times$ six intervals) within-subjects ANOVA. The results showed significant interval effects in almost all cases, confirming that responding changed over time, but there were only a few significant overall differences between average responding in all interwater intervals and appropriate portions of baseline.

To explore the difference from baseline responding at particular interwater intervals, we computed a chi-square value comparing responding under a particular interwater interval with responding in baseline at each relevant 4-sec bin. These chi-squares were summed over the bins in that particular interval (preserving the sign of the deviation from baseline), converted to $z$ scores, and summed over animals, following Cochran's (1954) procedure. All significant differences $(p<.05)$ between baseline and a particular interwater interval reported in the next two paragraphs are based on this procedure.

Figure 4 shows many similarities between the temporal distribution of responses around completed drinking bouts in baseline and the distribution of responses around individual ingestion opportunities under each interval constraint. For postdrinking responses such as wait-by-water, head-in-feeder, activity, nest, and run, the parallels in the shapes of the distributions of responding over time are sometimes remarkable. However, the animals did show a marked increase in concern with water. Both head-inwater and wait-by-water clearly exceeded baseline, especially just prior to water delivery and at the shorter interwater intervals. This increased focus on the water source was accompanied by a decrement in predrinking activity and nest at the shortest intervals and nest and run at the longest intervals. Also, animals were much less likely to be involved in food-related responses preceding and following ingestion than they were in baseline.

\section{The Categorization of Adjunctive Behavior}

The distinction between time-bound and proportional responses can be investigated by plotting the time from water ingestion to the peak (modal 4-sec bin) of responding for different responses as a function of the minimum interwater interval. At the smallest interwater intervals, the peak points lie on top of each other, but as the interval gets larger, the functions representing the peak points for interfood responses tend to fall into two broad classes. The peak-point bin either remains relatively constant across interwater intervals or shows a proportional increase as the interwater interval gets larger.

Figure 5 shows such a plot for the present data, excluding the 8-sec interval, for which many responses were absent and the peak points thus undefined. Rear and activity were relatively constant in peak points across all interwater intervals, except for a slight increase at the 256-

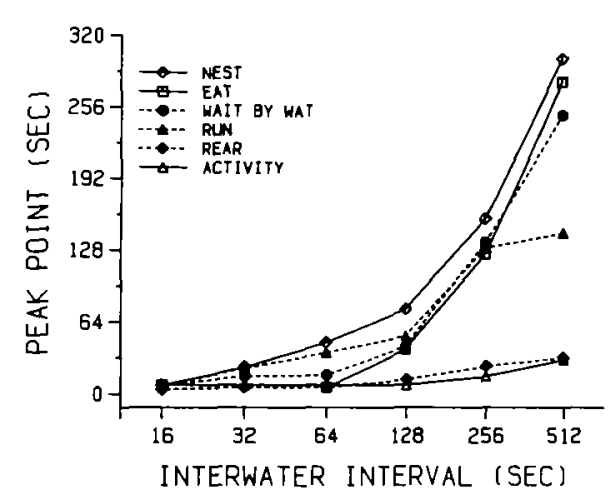

Figure 5. The mean time of peak responding for the different responses under each interwater interval condition. Notice that rear and activity are time-bound because they peak at approximately the same time following ingestion across most of the range of interwater intervals. Most other responses increase more proportionally.

and 512-sec intervals. At the other extreme, the peak points of nest, run, wait-by-water, and eat tended to increase as a direct function of the interwater interval. Notice that prior to the $128-\mathrm{sec}$ interval, eat was virtually nonexistent and wait-by-water was time-bound.

In short, several response changes occur at and following the interwater interval of $128 \mathrm{sec}$. Prior to that point, all responses except nest and run were either time-bound or absent. Beginning with $128 \mathrm{sec}$, wait-by-water and eat began to increase as a function of interwater interval, along with nest and run. It is worth noting that the peak points of these responses increased at approximately the same rate as the interwater interval from 128 to $512 \mathrm{sec}$ (except that run tailed off at the 512-sec interval).

\section{DISCUSSION}

The present results of presenting periodic access to water in a 24-h multiresponse environment generally supported and extended previous findings in shorter sessions and simpler environments (Carlisle et al., 1972; King, 1974; Myerson \& Christiansen, 1979; Reid et al., 1985; Wetherington \& Brownstein, 1979; Wetherington \& Riley, 1985). We found very few excessive responses, defined either by an increase over the daily total of responding in baseline or by an increase in the probability of responding per time-based opportunity during a drinking bout. With respect to daily totals, only wait-by-water and head-in-water showed marked increases, whereas activity showed a small, although consistent, increase. Both daily total feeding and drinking declined, and the remaining responses showed inconsistent effects across intervals and animals. With respect to responding per interdrink opportunity, only the predrink probability of wait-by-water and head-in-water markedly increased, whereas the predrink probabilities of activity, run, and nest decreased.

With respect to total responding within drinking bouts, the percentage of the daily response totals occurring within bouts increased for all responses as the interwater inter- 
val increased. At the 512 -sec interval, over $70 \%$ of the daily totals of most responses occurred within drinking bouts. When this information is combined with the total daily response data, it is obvious that most of the increased responding was related to redistribution of behavior from outside to inside drinking bouts. Only in the cases of waitby-water, head-in-water, and, to a lesser extent, activity, were the within-bout increases related to increases in daily response totals.

An interesting aspect of the redistribution of daily responding was the order in which responses showed withinbout increases. The first responses that increased were head-in-water and wait-by-water, followed by rear, activity, run, and, finally, by eat and nest. Increases in the latter responses, especially eat, occurred only as the time available to express the behavior outside the drinking bout and in the dark part of the light cycle became very small. It appears that rats are reluctant to eat during a drinking bout until the interwater interval is around $256 \mathrm{sec}$. Whether the increased eating at this point is due to the decreased time to eat outside the drinking bouts or to a momentary decrease in drinking motivation caused by the lengthened interval between drinks cannot be distinguished here. The data of Wetherington and Brownstein (1979) from 1-h sessions suggest that the interruption of a drinking bout by feeding can occur only because of the lengthening of the interwater interval.

The proportion of the interwater intervals (during a drinking bout) occupied by each behavior changed markedly across interwater intervals. At the 8-, 16-, and 32-sec intervals, most of the interval was taken up by head-inwater, wait-by-water, rear, and activity. During the longer intervals, run and nest increased in occurrence to over $10 \%$ of the intervals, whereas two initially dominant responses, activity and rear, decreased below run and nest in occurrence. The proportion of intervals with eating increased only at the 256- and 512-sec conditions.

Within-interval patterning was revealed by comparing the probabilities of responses preceding and following spaced individual drinks during each interwater interval against the baseline probabilities of these same responses preceding and following unconstrained drinking bouts. As indicated above, most responses decreased in probability with increasing interwater interval. In addition to the slight decreases in predrink probabilities of activity, run, and nest, the probabilities of eating and feeder-related responses were greatly reduced in both pre- and postdrink periods. The decline in food-related responses was most noticeable at interwater intervals below $128 \mathrm{sec}$, at which point food responding was nearly absent (see also Figure 3). At shorter intervals, eating occurred only when the animal was not in a drinking bout. At longer intervals, eating occurred within bouts, and its peak increased with the interval size.

Combining the plots of within-interval responding and peak responding across interwater intervals revealed three reasonably clear response classes: a time-bound class, including activity and rear; a relatively proportional class, including nest, eat, wait-by-water, and run; and a terminal class, including head-in-water. These behaviors correspond roughly to cyclic foraging sequences for water, which involve initial area-restricted search activity (headin-water, and rear), followed by the more general search and waiting responses of wait-by-water, nest, run, and, beginning with an interwater interval of $128 \mathrm{sec}$, eat. An increase in food-related responses (head-in-feeder and wait-by-feeder) accompanied the emergence of interwater eating. Finally, the focal search response of head-in-water predominated just before water delivery.

It is worth considering two exceptions to the picture presented by these findings. Bellingham et al. (1979) showed an increase in eating under a water-presentation schedule, and King (1974) showed an increase in wheelrunning under a water-presentation schedule. Both studies share two common characteristics. The responses that increased (eating in the first study and wheelrunning in the second) did not increase very much with respect to typical findings with periodic food in adjunctive paradigms (less than a factor of two). This suggests that different mechanisms may be at work with periodic food and water. Also, in the case of Bellingham et al. (1979), the increase in eating may have been judged inappropriately because it was compared with that of a control group for which there was less physical proximity between food and water.

The second common characteristic of both experiments is that both eating and running were unusually accessible responses, provided the animal focused its responding on the water source. In the case of Bellingham et al. (1979), each dipper full of water was presented in the middle of a tray filled with food pellets. Eating could have occurred almost as a by-product while the hungry animal was rooting and digging in the tray for water (Reberg, Mann, \& Innis, 1977). Based on the probable high frequency of prewater digging, we expect that a good deal of the increase in eating occurred pre- rather than postwater, a conclusion supported by the data of Bellingham et al. (1979), which showed less eating when there was a competing prewater barpress requirement.

In the case of King (1974), the wheel constituted the experimental space into which a drinking tube was inserted when water was made available, so that any postwater rearing or locomotor movements around the water location could be readily translated into the relatively small unit of half wheelturns. Also, based on the work of Gawley, Timberlake, and Lucas (1987), which used a similar apparatus and procedure, King's rats may have taken in less water than in baseline, resulting in a slight decrease in eating and a resultant increase in running during the experimental session. An inspection of King's data supports this view by showing such a decrease in water intake, but more decisive evidence can only come from knowledge of the temporal distribution of responding during the session.

\section{Comparison of Food and Water Efiects}

Excessiveness. An obvious similarity between the present results with periodic water and the results of Lucas 
et al. (1988) using periodic food was the marked increase in responses directed to the location where the restricted commodity was delivered. Head-in-feeder and wait-byfeeder or head-in-water and wait-by-water increased whether they were measured against total baseline responding or the conditional probability of responding around baseline ingestion bouts. Most, but not all, of the increase in conditional probability occurred in preingestion baseline and was accompanied by a decline in activity and nest at the short interreward intervals.

The major difference between food and water reward was that periodic food produced a highly active animal across the entire interingestion interval and periodic water did not. In the case of food reward, feeder-related responses, drinking-related responses, run, activity, and rear all became excessive at some intervals, compared with baseline totals. With water, only drinking-related responses increased much; eat decreased consistently, as did preingestion run at the longer intervals, and the remaining responses showed largely inconsistent effects. Reberg, Mann, and Innis (1977) showed similar differences in the behavior of pigeons on food versus water trials at a 15-sec interreward interval. Following food, pigeons roamed the chamber "down and back" in the next interreward interval. In contrast, following water, the pigeons remained close to the hopper.

An apparent contradiction of this difference in activity for food and water reward is that nest occupied considerably more of the interreward interval in the case of food. The explanation seems to be that with animals given periodic water, water-related investigation and waiting dominated all responses, including nest-related behavior as well as general activity. For animals given periodic food, activity, which included popping in and out of the nest for brief stays, took precedence.

Functional relations with reward frequency. Periodic water produced no bitonic relationships between reward frequency and total daily responding. In the case of periodic food, only head-in-feeder, wait-by feeder, drink, head-in-water, and wait-by-water showed an increase with interval size, followed by a drop-off at the 512 -sec interval (data from Table 2 of Lucas et al., 1988). As to the relationship between reward frequency and local response rate, no one form of relationship predominated. In the case of food, head-in-feeder and drinking both showed direct relationships with reward frequency, but the other responses showed bitonic or inverse relationships. In the case of water, both activity and rear showed a modest bitonicity, with a peak at the 32-sec interwater interval. Head-in-water showed a more or less direct relationship with frequency of reward, whereas the remaining responses, wait-by-water, eat, nest, and run, showed an inverse relationship to frequency of reward.

The data as a whole suggest that the key to both a bitonic relationship between reward frequency and total responding and a direct relationship between reward frequency and local response rate lies in the characteristics of the individual response and the degree of local compe- tition for expression. Responses that are time-bound in expression during postfood or postwater search appear to compete well for expression at the shortest interreward intervals but appear to be limited by self-terminating qualities and competition with more general search behaviors at longer intervals. These responses show a direct relationship between reward frequency and proportion of interval occupied and a bitonic relationship between reward frequency and total responding. Under food reward, all the responses related to eating and drinking showed both direct (local response rate) and bitonic (total responding) relationships with reward frequency. Under water reward, no responses showed this pattern.

For responses more related to general search behavior, such as activity or run, bitonic and direct relationships were infrequent. Instead, inverse relationships between frequency of reward and the measures of total responding and local response rate were common for both food and water. These responses apparently do not compete well with the time-bound responses at the shortest interreward intervals, but they have fewer self-terminating qualities and compete better for expression at the longer intervals. Many of what Staddon (1977) termed facultative responses fall into this category.

The temporal organization of responding. Another similarity between the effects of periodic food and water was in the temporal organization of postfood responses into three major classes based on their point(s) of peak responding and their conditional probabilities of responding pre- and postreward. One class showed a time-bound peak immediately following ingestion, a second class of responses showed increases in their point of peak responding directly related to the size of the interval, and a third response class showed a peak immediately prior to the delivery of reward. These classes appear to correspond to motivational states related to cyclic foraging, including postfood area-restricted search, more general search, and prefood focal search.

An obvious difference here is that periodic food additionally produced peaks of drinking and rearing partway between peak points for postfood and general search classes. The peaks of drinking and rearing were timebound at the shorter intervals but increased more proportionally at the longest intervals. There were no comparable behaviors under periodic water. Eating, the response that might be expected to increase if eating and drinking were reciprocally related, never appeared time-bound and rarely occurred prior to the 128 -sec interval.

The overall comparison of food and water data in the literature strongly suggests a relationship between feeding and drinking that varies asymmetrically with the deprivation level of the animal. From free-baseline data, we know that drinking both precedes and follows eating (Kissileff, 1969; Lucas, Timberlake, \& Gawley, 1989), suggesting the possibility of a reciprocal relationship in which drinking directly increases the motivation for (instigates) eating and eating instigates drinking. There is little question that eating instigates drinking, because large drink- 
ing bouts nearly always follow an eating bout. Eating, however, follows only a relatively small bout of drinking, not the larger postfood drinking bouts. Analyses by Lucas et al. (1989) suggest that the major function of preeating drink bouts is to reduce a state of thirst, thereby releasing the suppression of eating.

This interpretation fits well with the data from periodic reward delivery (Lucas et al., 1988). The prevalence of polydipsia under periodic food indicates that eating directly instigates drinking, although so long as food is almost continuously available (at a rate of better than one 94-mg pellet each $16 \mathrm{sec}$ ), rats will not interrupt eating to drink. In contrast, drinking bouts in relatively thirsty animals appear to suppress, rather than instigate, eating. Eating is almost completely absent until there is at least $128 \mathrm{sec}$ between drinks, and, even then, eating is well below what occurs following drinking under conditions of free food and water. The only exception to suppression appears to occur when mildly thirsty animals take a brief drink - an eating bout frequently follows (Kissileff, 1969; Lucas et al., 1989). In short, eating instigates drinking, and a relatively brief interruption of an eating bout allows drinking to occur. On the other hand, drinking, at best, permits or releases eating, and thirst actively suppresses eating, even when the animal is involved in a long and highly discontinuous bout of drinking.

\section{Conclusion}

By way of overall summary, the periodic presentation of either water or food produced several forms of responding at the beginning and end of the interingestion inter$\mathrm{val}$. These responses (primarily head-in-water or headin-feeder) were focused on the location of reward delivery. During the remainder of the interreward interval, periodic-food animals were highly active in drinking, rearing, activity, running, and nest entries, although animals also increased wait-by-feeder late in the interval. In contrast, periodic-water animals in the middle portion of longer interwater intervals primarily showed wait-bywater (although all animals also ate and nested at long interwater intervals, and 2 animals showed significant increases in total wheelrunning at medium-to-long interwater intervals). An inspection of video tapes of the rats' behavior revealed that the waiting responses also differed between food and water. In the case of food, there was considerable movement and investigation associated with waiting in and moving in and out of the reward quadrant. The latter occurred especially at the longer interreward times. In the case of water, the rats tended to remain in the reward quadrant at all intervals except $8 \mathrm{sec}$, and they spent much of this time motionless and/or lying down.

These differences between the effects of periodic food and periodic water on interingestion behavior in rats are found in other procedures as well. The presentation of food, but not water, appears to entrain active search behaviors, including locomotion and wheelrunning. Animals anticipating food by an hour or more engage in marked bouts of anticipatory activity and specific manipulation responses. Animals anticipating water show much less vigorous anticipatory behavior, often waiting for water in a single location (see Roper, 1984).

Taken together, these results suggest the view that interingestion responses are related to cycles of foraging (Timberlake, 1990; Timberlake \& Lucas, 1989). The three classes of behavior in the present study were read ily interpretable as postreward search, general search and wait, and prereward search. The relatively low level of activity in the present experiment compared with the results with food reward makes sense in such a framework. In the rat's niche, finding a single food item usually signals an increased probability of finding a similar item nearby. Thus, rats under periodic food delivery should show forms of search behavior covering the immediate location, followed by a broadening search for food in other areas as more time passes. In contrast, finding a small amount of water more often predicts recurrence of water at the identical location (as the local water table begins to fill in the amount removed). Finding more water by expanding the search may well be less likely than the chances of more water collecting at the initial location.

\section{REFERENCES}

Bellingham. W. P., Wayner, M. J., Barone. F. C. (1979). Schedule induced eating in water deprived rats. Physiology \& Behavior. 23. I 105-1107.

Campagnoni, F. R. lawler, C. P., Cohen, P. S. (1986). Tem poral patterns of reinforcer-induced general activity and attack in pigeons. Physiology of Behavior, 37, 577-582

Campell, B. A., Cicala, G. A. (1962). Studies of water depriva tion in rats as a function of age. Joumal of Comparative \& Physio logical Psychology, 55. 763-768.

Carlisle, H. I., Shanab, M. E., Simpson, C. W. (1972). Schaduleinduced behaviors: Effect of intermittent water reinforcement on food intake and body temperature. Psychonomic Science, 26, 35-36

Cochran. W. G. (1954). Some methods for strengthening the common $X^{2}$ tests. Biometrics, 10, 417-451

Cohen, P. S. Looney, T. A., Campagnoni, F. R., Lawier, C. P (1985). A two-state model of reinforcer-induced motivation. In $F$. $R$ Brush \& J. B. Overmier (Eds.), Affect, conditioning, and cognirion Essays on the determinants of behavior (pp. 281-297). Hillsdale, NJ Eribaum.

FALK, J. L. (1961). Production of polydipsia in normal rats by an in termittent food schedule. Science, 133, 195-196.

FALK, J. L. (1969). Conditions producing psychogenic polydipsia in animals. Annals of the New York Academy of Sciences, 9, 19-25.

FALK, J. L. (1971). The nature and determinants of adjunctive behavior Physiology \& Behavior, 6, 577-588.

FALK, J. L. (1977). The origin and functions of adjunctive behavior Animal Learning \& Behavior, 5, 325-335.

Gawlex, D. J., Timberlake, W., LuCas, G. A. (1987). System specific differences in behavior regulation: Overrunning and under drinking in molar nondepriving schedules. Joumal of Experimental Psychology: Animal Behavior Processes, 13, 354-365

HaLL, J, F. (1955). Activity as a function of a restricted drinking sched ule. Joumal of Comparative \& Physiological Psychology, 48, 265-266.

Killeen, P., Hanson, S. J., a Osbonne, S. R. (1978). Arousal: Its genesis and manifestation as response rate. Psychological Review, 85. $571-581$

KING, G. D. (1974). Wheel running in the rat induced by a fixed-time presentation of water. Animal Leaming \& Behavior, 2, 325-328. 
KISSILEFF, H. R. (1969). Food-associated drinking in the rat. Joumal of Comparative \& Physiological Psychology, 67, 284-300.

LeHner, P. (1979). Handbook of ethological methods. New York: Garland Press.

Lucas, G. A., Timberlake, W., Gawley, D. J. (1988). Adjunctive behavior of the rat under periodic food delivery in a 24-hour environment. Animal Leaming \& Behavior, 16, 19-30.

Lucas, G. A., Timberlake, W., \& Gawley, D. J. (1989). Learning and meal associated drinking: Adjustments in postprandial drinking following meal related deficits. Physiology \& Behavior, 46, 361-367.

MCDonald, G. E., DE Toledo, L. (1974). Partial reinforcement effects and type of reward. Learning \& Motivation, 5, 288-298.

Myerson, J., \&hristiansen, B. (1979). Temporal control of eating on periodic water schedules. Physiology \& Behavior, 23, 279-282.

Petersen, M. R., Lyon, D. O. (1978). Schedule-induced polydipsia in rats living in an operant environment. Joumal of the Experimental Analysis of Behavior, 29, 493-503.

RACHLin, H., KRASNoff, J. (1983). Eating and drinking: An economic analysis. Journal of the Experimental Analysis of Behavior, 39. 385-404.

Reberg, D., MANN, B., \& INNIS, W. K. (1977). Superstitious behavior for food and water in the rat. Physiology \& Behavior, 19, 803-806

Reid, A. K., Staddon, J. E. R. (1986). Mechanisms of schedule entrainment. In S. J. Cooper \& C. T. Dourish (Eds.), The neurobiology of behavioural stereotypy. London: Oxford University Press.

Reid, A. K., VAzQuez, P. P., Rico, J. A. (1985). Schedule induction and the temporal distributions of adjunctive behavior on periodic water schedules. Animal Learning \& Behavior, 13, 321-326.

Riley, A. K., \& Wetherington, C. L. (1989). Schedule-induced polydipsia: Is the rat a small furry human? (An analysis of an animal model of human alcoholism). In S. B. Klein \& R. R. Mowrer (Eds.), Contemporary learning theories: Instrumental conditioning theory and the impact of biological constraints on leaming (pp. 205-233). Hillsdale, NJ: Erlbaum.

ROPER, T. J. (1981). What is meant by the term "schedule-induced," and how general is schedule induction? Animal Learning \& Behavior, 9, 433-440.
Roper, T. J. (1984). Response of thirsty rats to absence of water: Frustration, disinhibition or compensation? Animal Behaviour, 32, 1225-1235

Staddon, J. E. R. (1977). Schedule-induced behavior. In W. K. Honig \& J. E. R. Staddon (Eds.), Handbook of operant behavior (pp. 125152). New York: Prentice-Hall.

Staddon, J. E. R., \&immelhag, V. (1971). The "superstition" experiment: A reexamination of its implications for the principles of adaptive behavior. Psychological Review, 78, 3-43.

Timberlake, W. (1982). Controls and schedule-induced behavior. Animal Learning \& Behavior, 10, 535-536.

TimberLaKe, W. (1990). Natural learning in laboratory paradigms. In D. A. Dewsbury (Ed.), Contemporary issues in comparative psychology (pp. 31-54). Sunderland, MA: Sinauer Associates.

Timberlake, W., LuCAS, G. A. (1989). Behavior systems and learning: From misbehavior to general laws. In S. B. Klein \& R. R. Mowrer (Eds.), Contemporary learning theories: Instrumental conditioning theory and the impact of biological constraints on learning (pp. 237275). Hillsdale, NJ: Erlbaum.

WETHERINGTON, C. L. (1979). Schedule-induced drinking: Rate of food delivery and Herrnstein's equation. Journal of the Experimental Analysis of Behavior, 32, 323-333.

WETHERINGTON, C. L. (1982). Is adjunctive behavior a third class of behavior? Neuroscience \& Biobehavioral Reviews, 6, 329-350.

Wetherington, C. L., \& Brownstein, A. J. (1979). Schedule control of eating by fixed-time schedules of water presentation. Animal Learning \& Behavior, 7, 38-40.

Wetherington, C. L., Riley, A. L. (1985). Differences in food consumption under intermittent and continuous reinforcement schedules of water delivery: Some implications for schedule-induced behavior. Animal Learning \& Behavior, 13, 331-337.

(Manuscript received July 6, 1990 ; revision accepted for publication June 24, 1991.) 